PROCEEDINGS OF THE AMERICAN MATHEMATICAL SOCIETY

Volume 124, Number 9, September 1996

\title{
AUTOMATIC REALIZABILITY OF GALOIS GROUPS OF ORDER 16
}

\author{
HELEN G. GRUNDMAN AND TARA L. SMITH
}

(Communicated by Lance W. Small)

\begin{abstract}
This article examines the realizability of small groups of order $2^{k}, k \leq 4$, as Galois groups over arbitrary fields of characteristic not 2. In particular we consider automatic realizability of certain groups given the realizability of others.
\end{abstract}

\section{INTRODUCTION}

In this article we are concerned with automatic realizability results for small Galois 2-groups, particularly those of order 16 . We ask when it is true that the realizability of a particular group $G$ as a Galois group over a field $K$ implies the realizability of another group $H$ as a Galois group over $K$. In some cases, these results hold over all fields. In others, the automatic realizability depends on specific properties of $K$. We are also interested in field-theoretic criteria which guarantee the realizability of certain groups. By a famous result of Witt [Wi:1936], Satz, p.237, the realizability of a 2 -group $G$ over a field of characteristic 2 depends only on the minimal number of generators of $G$. For this reason we will always assume our fields to be of characteristic not 2 .

We obtain our results primarily by considering the obstructions to the realizability of the groups, expressed in terms of products of quaternion algebras in the Brauer group $\operatorname{Br}(K)$ of the base field $K$. By manipulating these expressions, one can often show that triviality of the obstruction for one group implies triviality of the obstruction for another. This is particularly true if additional information on the field, such as its level, is taken into consideration. The obstructions for these groups have appeared in various references. A complete survey of known results is provided in [GSS:1995]. We quote these results below and use them heavily in this article. Realizability questions have been considered previously in the two articles by C. U. Jensen, [Je:1989] and [Je:1992]. Jensen considers the structure of the groups explicitly in most instances, and often describes precisely how to construct fields realizing the groups, whereas our approach is less constructive. He also works

Received by the editors September 20, 1994 and, in revised form, March 6, 1995 .

1991 Mathematics Subject Classification. Primary 12F10, 12F12.

The first author's research was supported in part by National Science Foundation Grant No. DMS-9115349 and the Alice Lee Hardenbergh Clark Faculty Research Grants Fund of Bryn Mawr College. The second author's research was supported in part by the National Security Agency and the Taft Memorial Fund of the University of Cincinnati.

(C)1996 American Mathematical Society 
with finite 2-groups in general, whereas we work just with groups of order at most 16. We obtain a number of new realizability results by our methods.

Our goal is to systematically examine the groups of order 16 , and what can be said about the realizability of each group as a Galois group over fields of characteristic not 2. There are fourteen different groups of order 16, five of which are abelian. We let $C_{n}$ denote the cyclic group of order $n$. The five abelian groups are $C_{16}, C_{8} \times C_{2}, C_{4}^{2}, C_{4} \times C_{2}^{2}$, and $C_{2}^{4}$. By known results ([Wh:1957], [KuLe:1975], [GSS:1995]) realizability for these groups is determined solely by realizability of $C_{4}$ and the size of the square class group $\left|\dot{K} / \dot{K}^{2}\right|$. Note that the criterion for the realizability of $C_{4}^{2}$ over $K$ is simply the existence of two $C_{4}$-extensions containing distinct quadratic subfields. There are also the two decomposable groups obtained by taking the direct products of each of the two nonabelian groups of order 8 , the dihedral group and the quaternion group, with $C_{2}$; we denote these two groups as $D_{8} \times C_{2}$ and $Q_{8} \times C_{2}$ respectively. Again, it is easy to see that $D_{8} \times C_{2}$ (respectively $\left.Q_{8} \times C_{2}\right)$ is realizable if and only if $D_{8}$ (respecitively $Q_{8}$ ) is realizable, and $\left|\dot{K} / \dot{K}^{2}\right|$ is large enough; see [GSS:1995]. (We write $D_{2 n}$ to denote the dihedral group of order $2 n$.)

We introduce notation and presentations for each of the remaining seven indecomposable nonabelian groups below. We then give a list of the obstructions for each of the ten groups in which we will be interested. In $\S 2$ we give group-by-group descriptions of the results we have obtained for realizability, providing proofs for those results that are not widely known. In $\S 3$ we present results in terms of conditions on the level of the base field. Our results agree with or extend those obtained in [Je:1989], [Je:1992] for groups of order 16. Similar results have also recently been obtained in [L:1995].

The seven indecomposable nonabelian groups of order 16 are: $D C$, the central product of $D_{8}$ with $C_{4}$ amalgamating the central elements of order $2 ; Q \curlywedge C$, the pullback of the system $\left(Q_{8} \rightarrow C_{2}, C_{4} \rightarrow C_{2}\right)$ and also a semidirect product $C_{4} \rtimes C_{4}$; $D \curlywedge C$, the pullback of the system $\left(D_{8} \rightarrow C_{2}, C_{4} \rightarrow C_{2}\right)$ and also a semidirect product $\left.\left(C_{4} \times C_{2}\right) \rtimes C_{2}\right) ; D_{16}$, the dihedral group of order $16 ; Q_{16}$, the quaternion group of order 16; $S D_{16}$, the semidihedral or quasidihedral group of order 16; and $M_{16}$, the modular group of order 16 . Their presentations are as follows:

$$
\begin{gathered}
D C \cong\left\langle x, y, z \mid x^{2}=y^{2}=z^{4}=1, y^{-1} x y=x z^{2},[x, z]=[y, z]=1\right\rangle, \\
Q \curlywedge C \cong\left\langle x, y \mid x^{4}=y^{4}=1, y^{-1} x y=x^{-1}\right\rangle, \\
D \curlywedge C \cong\left\langle x, y, z \mid x^{4}=y^{2}=z^{2}=1, y^{-1} x y=x z,[x, z]=[y, z]=1\right\rangle, \\
D_{16} \cong\left\langle x, y \mid x^{8}=y^{2}=1, y^{-1} x y=x^{-1}\right\rangle, \\
Q_{16} \cong\left\langle x, y \mid x^{8}=y^{4}=1, y^{-1} x y=x^{-1}, y^{2}=x^{4}\right\rangle, \\
S D_{16} \cong\left\langle x, y \mid x^{8}=y^{2}=1, y^{-1} x y=x^{3}\right\rangle, \\
M_{16} \cong\left\langle x, y \mid x^{8}=y^{2}=1, y^{-1} x y=x^{5}\right\rangle .
\end{gathered}
$$

The following proposition is a compilation of all of the obstructions for the realizability of the ten groups we are interested in, taken from [GSS:1995]. Details and additional references can be found in that article.

Proposition 1. (1) A necessary and sufficient condition for the realizability of the group $C_{4}$ as a Galois group over the field $K$ is the existence of an element 
$a \in \dot{K}-\dot{K}^{2}$ such that $(a,-1)=1 \in B r_{2}(K)$. In this case, the group $C_{4}$ can be realized by an extension of $K$ which is quadratic over $K(\sqrt{a})$.

(2) A necessary and sufficient condition for the realizability of the dihedral group $D_{8}$ of order 8 as a Galois group over the field $K$ is the existence of independent elements $a, b \in \dot{K} / \dot{K}^{2}$ such that $(a, b)=1 \in B r_{2}(K)$. In this case, the group $D_{8}$ is realized by an extension of $K$ which is cyclic over $K(\sqrt{a b})$.

(3) A necessary and sufficient condition for the realizability of the group $Q_{8}$ as a Galois group over the field $K$ is the existence of independent elements $a, b \in \dot{K} / \dot{K}^{2}$ such that $(a, b)(a b,-1)=1 \in B r_{2}(K)$.

(4) A necessary and sufficient condition for the realizability of the group DC as a Galois group over the field $K$ is the existence of independent elements $a, b, c \in$ $\dot{K} / \dot{K}^{2}$ such that $(a, b)(c,-1)=1 \in B r_{2}(K)$.

(5) A necessary and sufficient condition for the realizability of the group $Q \curlywedge C$ as a Galois group over the field $K$ is the existence of independent elements $a, b \in \dot{K} / \dot{K}^{2}$ such that $(a,-1)=(a, b)(b,-1)=1 \in B r_{2}(K)$.

(6) A necessary and sufficient condition for the realizability of the group $D \curlywedge C$ as a Galois group over the field $K$ is the existence of independent elements $a, b \in \dot{K} / \dot{K}^{2}$ such that $(a,-1)=(a, b)=1 \in B r_{2}(K)$.

(7) A necessary and sufficient condition for the realizability of the group $D_{16}$ as a Galois group over the field $K$ is the existence of independent elements a, $b \in \dot{K} / \dot{K}^{2}$ and an element $x \in \dot{K}$ such that $(a, b)=(a, 2)(x,-a b)=1 \in B r_{2}(K)$.

(8) A necessary and sufficient condition for the realizability of the group $Q_{16}$ as a Galois group over the field $K$ is the existence of independent elements $a, b \in \dot{K} / \dot{K}^{2}$ and an element $x \in \dot{K}$ such that $(a, b)=(a, 2)(x,-a b)(a b,-1)=1 \in B r_{2}(K)$.

(9) A necessary and sufficient condition for the realizability of the group $S D_{16}$ as a Galois group over the field $K$ is the existence of independent elements $a, b \in \dot{K} / \dot{K}^{2}$ and an element $x \in \dot{K}$ such that $(a, b)=(a,-2)(x,-a b)=1 \in B r_{2}(K)$.

(10) A necessary and sufficient condition for the realizability of the group $M_{16}$ as a Galois group over the field $K$ is the existence of independent elements $a, b \in \dot{K} / \dot{K}^{2}$ and an element $x \in \dot{K}$ such that $(a,-1)=(a, 2 b)(x,-1)=1 \in B r_{2}(K)$.

\section{REALIZABILITY RESUlTS FOR SPECIFIC GROUPS}

We begin this section with some definitions, and then proceed to examine results on realizability of each of the ten groups $C_{4}, D_{8}, Q_{8}, D C, Q \curlywedge C, D \curlywedge C, D_{16}, Q_{16}$, $S D_{16}$, and $M_{16}$. We use the obstructions provided in Proposition 1 as our criteria for realizability.

Definition. The level $s(K)$ of the field $K$ is the least positive integer $n$ such that -1 can be expressed as a sum of $n$ squares; $s(K)=\infty$ if no such integer exists. If $s(K)$ is finite, then $s(K)$ is a power of 2 . If $s(K)=\infty, K$ is formally real.

Definition. The field $K$ is pythagorean if every element which is a sum of squares is in fact a square. In this case either $s(K)=1$ and $K$ is quadratically closed, or $s(K)=\infty$.

Definition. An element $a \in \dot{K}-\dot{K}^{2}$ is rigid if the set of elements in $K$ represented by the quadratic form $\langle 1, a\rangle$ is precisely $\dot{K}^{2} \cup a \dot{K}^{2}$. The field $K$ is said to be rigid if every $a \in \dot{K}- \pm \dot{K}^{2}$ is rigid. Notice that if $K$ is rigid and $s(K)=2$, then $2 \in \pm \dot{K}^{2}$, and if $K$ is rigid and $s(K)=\infty$, then $2 \in \dot{K}^{2}$. 
Proposition 2.1. The group $C_{4}$ is realizable as a Galois group over $K$ if and only if $K$ is not pythagorean.

Proof. The obstruction to the realizability of $C_{4}$ is equivalent to the existence of an element $a \in K$ which is a sum of two squares but not a square.

Proposition 2.2. Assume $\left|\dot{K} / \dot{K}^{2}\right| \geq 4$. The dihedral group $D_{8}$ of order 8 is always realizable over $K$ unless $s(K)=1$ and $K$ is rigid.

Proof. If $s(K) \geq 2$ and $\left|\dot{K} / \dot{K}^{2}\right| \geq 4$, then there exists $a \in \dot{K}$ such that $a,-a$ are independent $\bmod \dot{K}^{2}$. Since $(a,-a)=1 \in B r_{2}(K)$ always, we see that $D_{8}$ is realizable. If $s(K)=1$ and there do not exist $a, b \in \dot{K}$, independent $\bmod \dot{K}^{2}$, such that $(a, b)=1 \in B r_{2}(K)$, then for all $a \in \dot{K}-\dot{K}^{2}$ we must have that the form $\langle 1, a\rangle$ only represents elements in $\dot{K}^{2} \cup a \dot{K}^{2}$. Thus $K$ is rigid.

Proposition 2.3. Assume $\left|\dot{K} / \dot{K}^{2}\right| \geq 4$. The quaternion group $Q_{8}$ of order 8 is always realizable over $K$ unless either $s(K)=\infty$ and sums of squares are rigid in $K$, or $s(K)=1$ and $K$ is rigid. In particular, if $K$ is not formally real, then $Q_{8}$ is realizable if and only if $D_{8}$ is realizable. If $Q_{8}$ is realizable, then $C_{4}$ is realizable.

Proof. The proof of all but the last statement is contained in [Wa:1990] and can be derived from Proposition 1 using the equality $(a, b)(a b,-1)=(-a,-b)(-1,-1)$ in $\mathrm{Br}_{2}(F)$. The last statement follows by noting that the given conditions for realizability of $Q_{8}$ imply $K$ cannot be pythagorean.

Proposition 2.4. Assume $\left|\dot{K} / \dot{K}^{2}\right| \geq 8$. The group DC is realizable over $K$ unless $K$ is rigid, in which case $s(K)=1,2$, or $\infty$.

Proof. The result is derived by manipulating the obstruction $(a, b)(c,-1)$ (see [MiSm:1991]).

We now consider realizability of $Q \curlywedge C$. Notice first that if $s(K)=1$, the obstruction for realizability of $Q \curlywedge C$ reduces to that for $Q_{8}$ (and for $D_{8}$ ). If $s(K)=$ 2 , let $a=-1$, and choose $b$ independent of -1 . Then $(-1,-1)=1 \in B r_{2}(K)$ and $(-1, b)(b,-1)=1$, so there is a $Q \curlywedge C$-extension of $K$. The following proposition considers realizability of $Q \curlywedge C$ in general, giving an automatic realizability result that holds over any field $K$.

Proposition 2.5. The group $Q \curlywedge C$ is realizable over $K$ if and only if $Q_{8}$ is realizable over $K$.

Proof. If $Q \curlywedge C$ is realizable, then clearly so is $Q_{8}$, as well as $C_{4}$, since they are quotients. Conversely, assume $Q_{8}$ is realizable. Then we have independent $a, b$ such that $(a, b)(a,-1)(b,-1)=1 \in B r_{2}(K)$. By manipulation of quaternion algebras we have $(-a,-b)(-1,-1)=1$, and working with known criteria for the splitting of a tensor product of two quaternion algebras, we have $\langle a, b, a b\rangle \simeq\langle 1,1,1\rangle$, so $a$ is a sum of three squares, but not a square. If $a$ is a sum of two squares, then $(a,-1)=1$, giving the desired $Q \curlywedge C$-extension. If not, then there exists $c \in K$ which is a sum of two squares but not a square, such that $a$ is represented by the form $\langle 1, c\rangle$, so $\langle 1, c\rangle \simeq\langle a, a c\rangle$. Then $(a,-c)=1 \in B r_{2}(K)$, so $(a, c)(a,-1)=1$, and since $c$ is a sum of two squares, $(c,-1)=1$. Now $a, c$ are independent since $c$ is a sum of two squares but $a$ is not, so we have a $Q \curlywedge C$-extension of $K$. 
Proposition 2.6. Assume $\left|\dot{K} / \dot{K}^{2}\right| \geq 4$. The group $D \curlywedge C$ is realizable over $K$ unless (a) $s(K)=1$ and $K$ is rigid, (b) $s(K)=\infty$ and $K$ is pythagorean, or (c) $s(K)=2$ and $C_{4} \times C_{4}$ is not realizable as a Galois group over $K$.

Proof. If $s(K)=1$, the obstruction for $D \curlywedge C$ reduces to that for $D_{8}$, and the group is not realizable precisely when $K$ is rigid (condition (a)). If $s(K) \geq 2$ and there exists $a \neq-1 \in \dot{K}$ which is a sum of two squares but not a square, then $(a,-1)=(a,-a)=1 \in B r_{2}(K)$, satisfying the obstruction to the realizability of $D \curlywedge C$ as a Galois group over $K$. If there is no such $a$, then either all sums of squares are in fact squares, in which case $K$ is pythagorean and formally real (condition (b)), or the only element $a \in \dot{K}-\dot{K}^{2}$ for which $(a,-1)=1$ is $a=-1$, in which case $s(K)=2$ and $C_{4} \times C_{4}$ cannot be realized as a Galois group over $K$ (condition (c)). Note that in condition (c), the only $C_{4}$-extensions of $K$ will be those lying over $K(\sqrt{-1})$.

When $s(K)=1$, all the nonabelian groups of order 16 and exponent 4 are realizable if any of them are, provided $\left|\dot{K} / \dot{K}^{2}\right|$ is large enough. This is clear from the obstructions given in Proposition 1, since the obstructions for all of these groups become identical when -1 is a square in $K$. We have the following result.

Corollary 2.7. If $s(K)=1$, then any one of the groups $D_{8}, Q_{8}, Q \curlywedge C, D \curlywedge C$ is realizable over $K$ if and only if the other three groups are realizable over $K$. If $\left|\dot{K} / \dot{K}^{2}\right| \geq 8$, the realizability of these groups is also equivalent to the realizability of $D C$.

The following proposition links realizability of $D \curlywedge C$ with that of $D C$ when $s(K) \geq 2$. Combining this with Corollary 2.7 shows that $D \curlywedge C$ automatically realizes $D C$ if $K$ has enough square classes.

Proposition 2.8. Assume $s(K) \geq 2$ and $\left|\dot{K} / \dot{K}^{2}\right| \geq 8$. If $2 \in-\dot{K}^{2}$, then realizability of $D \curlywedge C$ implies $D_{16}$ is realizable, which implies $D C$ is realizable. If $2 \in \dot{K}^{2}$, then realizability of $D \curlywedge C$ implies $S D_{16}$ is realizable, which implies $D C$ is realizable. If $2 \notin \pm \dot{K}^{2}, D C$ is realizable.

Proof. First observe that if $2 \notin \pm \dot{K}^{2}$, then since the form $\langle 1,-2\rangle$ represents -1 , $K$ cannot be rigid and $D C$ is realizable. Now assume $D \curlywedge C$ is realizable. We may fix $a, b$ independent $\bmod \dot{K}^{2}$ such that $(a,-1)=(a, b)=1 \in B r_{2}(K)$. First assume $2 \equiv-1 \bmod \dot{K}^{2}$. In the obstruction for the realizability of $D_{16}$, choose the same $a, b$ and let $x=1$. Then the obstruction for $D_{16}$ becomes $(a, b)$ and $(a, 2)(1,-a b)=(a,-1)$, both of which we have assumed to be trivial. Thus $D_{16}$ is realizable. Now assume $2 \equiv-1 \bmod \dot{K}^{2}$ and $D_{16}$ is realizable. Then there exist $a, b$ independent and $x \in \dot{K}$ such that $(a, b)=(a,-1)(x,-a b)=1$. If $a \neq-b \in \dot{K} / \dot{K}^{2}$, then $a, b,-1$ are independent; and since $s(K)=2$ (because $2 \equiv-1$ ), we have $(a, b)(-1,-1)=1$, realizing $D C$. If $a=-b$, then we have $(a,-1)=1$. Choose $c \in \dot{K}$ such that $a, c,-1$ are independent. Then $(c,-c)(a,-1)=1$, realizing $D C$.

For the remainder of the proof, assume $2 \in \dot{K}^{2}$. Then the criterion for the realizability of $S D_{16}$ is the existence of independent $c, d \in \dot{K}$ and $x \in \dot{K}$ such that $(c, d)=(c,-1)(x,-c d)=1$. Choose $c=a, d=b$, where $(a,-1)=(a, b)=1$ is given by the realizability of $D \curlywedge C$, and take $x=1$.

We show below (Proposition 2.15) that, in general, realizability of $S D_{16}$ implies realizability of $C_{4}$. If $s(K) \geq 4, C_{4}$ automatically realizes $D C$. If $s(K)=2$, realizability of $S D_{16}$ gives us independent $a, b$ such that $(a, b)=(a,-1)(x,-a b)=1$ 
for some $x \in \dot{K}$. If $a=-b$, then $(a,-1)=1$. Choose $c \in \dot{K}$ such that $a, c,-1$ are independent. Then $(c,-c)(a,-1)=1$, realizing $D C$. If $a \neq-b$, then $a, b,-1$ are independent, and $(a, b)(-1,-1)=1$, since $s(K)=2$, so we again obtain the realizability of $D C$.

Proposition 2.9. Assume $\left|\dot{K} / \dot{K}^{2}\right| \geq 4$. The group $D_{16}$ is always realizable over $K$ if $s(K) \geq 4$. If $s(K)=2$, then $D_{16}$ is realizable unless $2 \in-\dot{K}^{2}$, in which case realizability of $D \curlywedge C$ implies realizability of $D_{16}$. If $s(K)=1$, any one of the groups $D_{16}, Q_{16}$, and $S D_{16}$ is realizable if and only if the other two are, and realizability of any of these groups implies the realizability of $D_{8}$. This in turn implies the realizability of $M_{16}, Q_{8}, Q \curlywedge C$, and $D \curlywedge C$, and if $\left|\dot{K} / \dot{K}^{2}\right| \geq 8$, of $D C$ as well. Thus if $s(K)=1$ and $\left|\dot{K} / \dot{K}^{2}\right|$ is large enough, $D_{16}$ automatically realizes all other groups of order 16 .

Proof. The criterion for the realizability of $D_{16}$ is the existence of independent $a, b \in \dot{K}$, and $x \in \dot{K}$, such that $(a, b)=(a, 2)(x,-a b)=1 \in B r_{2}(K)$. Assume first $s(K) \geq 2$. If $2 \notin \pm \dot{K}^{2}$, choose $a=-2, b=2$; then $D_{16}$ is realizable. If $2 \in \dot{K}^{2}$, choose $a=-b$ for some $a \notin \pm \dot{K}^{2}$. If $2 \in-\dot{K}^{2}$, then necessarily $s(K)=2$, and $D \curlywedge C$ realizes $D_{16}$ as shown in Proposition 2.8.

Assume now that $s(K)=1$. The realizability criteria for all three groups $D_{16}, Q_{16}, S D_{16}$ reduce to the existence of independent $a, b \in \dot{K}$ and $x \in \dot{K}$ such that $(a, b)=(a, 2)(x, a b)=1 \in B r_{2}(K)$. Thus they are all equivalent and clearly imply the realizability of $D_{8}$. Realizability of $D_{8}$ implies the realizability of the other groups listed, as shown in Corollary 2.7 above for all groups except $M_{16}$, and in Corollary 2.14 below for $M_{16}$. When $s(K)=1,(a, a)=1$ for all $a \in \dot{K}$, realizing $C_{4}$, so realizability of $D_{16}$ is sufficient to guarantee the realizability of all groups of order 16 over $K$ when $\left|\dot{K} / \dot{K}^{2}\right|$ is large enough.

Corollary 2.10. If $s(K)=1$ and $2 \in \dot{K}^{2}$, then the realizability of any one of the groups $D_{8}, Q_{8}, D \curlywedge C, Q \curlywedge C, D_{16}, Q_{16}, S D_{16}, M_{16}$ implies the realizability of all of the others.

Proof. Under the given assumptions on $K$, we have already seen the equivalence of the realizability criteria for the groups $D_{16}, Q_{16}$, and $S D_{16}$ (Proposition 2.9), and for the groups $D_{8}, Q_{8}, Q \curlywedge C, D \curlywedge C$, (Corollary 2.7), and the result for $M_{16}$ follows from Corollary 2.14 below. Also $D_{8}$ is a quotient of $D_{16}$, so is trivially realized by it. Thus, what remains to show is that realizability of $D_{8}$ implies realizability of $D_{16}$. If $-1,2 \in \dot{K}^{2}$, the criterion for $D_{16}$ becomes $(a, b)=(x,-a b)=1$ for some independent $a, b \in \dot{K}$ and some $x \in \dot{K}$. Choosing $x=1$ makes $D_{16}$ realizable whenever $D_{8}$ is.

Just as realizability of $Q_{8}$ implies realizability of $D_{8}$, so does realizability of $Q_{16}$ imply realizability of $D_{16}$, as the following proposition, together with Proposition 2.9 , shows.

Proposition 2.11. If $s(K) \leq 2, Q_{16}$ is realizable if and only if $D_{16}$ is. If $s(K) \geq 4$, realizability of $Q_{16}$ implies realizability of $C_{4}, D_{8}, D \curlywedge C, D_{16}, S D_{16}$, and $M_{16}$, and also of $D C$ if $\left|\dot{K} / \dot{K}^{2}\right| \geq 8$. If $2 \in \dot{K}^{2}$, realizability of $Q_{16}$ is equivalent to realizability of $Q_{8}$.

Proof. We have already seen in Proposition 2.9 that for $s(K)=1, Q_{16}$ is realizable over $K$ if and only if $D_{16}$ is. If $s(K)=2$ and either group is realizable, then we have 
independent $a, b \in \dot{K} / \dot{K}^{2}$ with $(a, b)=1$. If $a=-b$, then $(a, 2)=(a, 2)(-1,-1)$, and so realizability of either group implies that of the other. If $a \neq-b$, then $(a, 2)(x,-a b)=(a, 2)(x,-a b)(-1,-1)=(a, 2)(x,-a b)(-1,-a b)(-1, a b)=$ $(a, 2)(-x,-a b)(-1, a b)$, so the obstruction for $D_{16}$ becomes the obstruction for $Q_{16}$ by replacing $x$ with $-x$.

For the remainder of the proof, we assume $s(K) \geq 4$. The groups $D_{8}$ and $D_{16}$ are always realizable if $\left|\dot{K} / \dot{K}^{2}\right| \geq 4$. If $2 \notin \dot{K}^{2}$, then we have seen that the groups $C_{4}, D \curlywedge C$, and, if $\left|\dot{K} / \dot{K}^{2}\right| \geq 8$, also $D C$ are always realizable. To see that $S D_{16}$ and $M_{16}$ are realizable, note that $s(K) \geq 4$ and $2 \notin \dot{K}^{2}$ imply that $2,-2$ are independent. So taking $a=2, b=-2$, and $x=1$, the obstructions for $S D_{16}$ and $M_{16}$ are each trivial. Thus we now assume $2 \in \dot{K}^{2}$.

First we show realizability of $Q_{16}$ is equivalent to realizability of $Q_{8}$. Given $a, b$ realizing $Q_{16}$, we cannot have $a=-b$, since then $(-1,-1)=1$, implying $s(K) \leq 2$. Thus we have $(a, b)=(x,-a b)(a b,-1)$ for some $x \in \dot{K}$. If $x \in \dot{K}^{2}$, then $(a b,-1)=1$, so $(a, b)(a b,-1)=1$, realizing $Q_{8}$. If $x a b \in \dot{K}^{2}$, then $(x,-a b)=$ $(x,-x)=1$, so $(a b,-1)=1$, and we again have $Q_{8}$ as above. Finally, if neither $x$ nor $x a b$ is a square, then $x, a b$ are independent. Further, $(x, a b)(x a b,-1)=$ $(x, a b)(x,-1)(a b,-1)=(x,-a b)(a b,-1)=1$, realizing $Q_{8}$. Conversely, given $Q_{8}$ as a $K$-Galois group, we know we also have $Q \curlywedge C$ as a $K$-Galois group. Thus, there exist $a, b$ independent such that $(a,-1)=(a, b)(b,-1)=1$. Choose $c=a b, d=$ $b, x=a$. Then $(c, d)=(a b, b)=(a, b)(b,-1)=1$ and $(c, 2)(x,-c d)(c d,-1)=$ $(a,-1)=1$, and so we have a $Q_{16}$-extension of $K$.

It remains to show that when $2 \in \dot{K}^{2}$, realizability of $Q_{16}$ implies that of $C_{4}$, $D \curlywedge C, S D_{16}$, and $M_{16}$. We have seen that $Q_{16}$ realizes $Q \curlywedge C$, so we get $C_{4}$ because it is a quotient of $Q \curlywedge C$. Since $s(K) \geq 4, C_{4}$ is realizable if and only if $D \curlywedge C$ is realizable (Propositions 2.1 and 2.6). Suppose $(a,-1)=1$ realizes $C_{4}$. Necessarily $a$ and -1 are independent, since if $a=-1, s(K) \leq 2$. Let $b=-a$, $x=1$ in the obstruction for $M_{16}$. Then we have $(a,-1)=(a,-a)(1,-1)=1$, so $M_{16}$ is realizable. To see realizability of $C_{4}$ implies realizability of $S D_{16}$, again let $b=-a$. Then under the assumption $2 \in \dot{K}^{2},(a,-a)=(a,-1)=1$, and so $S D_{16}$ is realizable.

Proposition 2.12. If $s(K)=1$, then $S D_{16}$ is realizable if and only if $D_{16}$ and $Q_{16}$ are. If $s(K) \geq 2$ and $\left|\dot{K} / \dot{K}^{2}\right| \geq 4$, then $S D_{16}$ is always realizable unless $2 \in \dot{K}^{2}$, in which case realizability of $S D_{16}$ is implied by that of $D \curlywedge C$.

Proof. We have already seen in Proposition 2.9 that if $s(K)=1$, then $S D_{16}$ is realizable if and only if $D_{16}$ and $Q_{16}$ are. Also we have seen in Proposition 2.8 that if $s(K) \geq 2,2 \in \dot{K}^{2}$, and $D \curlywedge C$ is realizable, then so is $S D_{16}$. So it remains to show that if $2 \notin \dot{K}^{2}$ and $s(K) \geq 2$, we can always realize $S D_{16}$. If $2 \notin-\dot{K}^{2}$, then $2,-2$ are independent, and we can let $a=2, b=-2$; then $(2,-2)=1$, so the obstruction for $S D_{16}$ is trivial. If $2 \in-\dot{K}^{2}$, then necessarily $s(K)=2$. Choose $a \notin \pm \dot{K}^{2}$, and let $b=-a$. Then $(a,-2)=1$, so again $S D_{16}$ is realizable.

Proposition 2.13. Assume $\left|\dot{K} / \dot{K}^{2}\right| \geq 4$. The group $M_{16}$ is always realizable over $K$ unless either $s(K)=1$ and $K$ is rigid, or $s(K)=\infty$ and $K$ is pythagorean. Thus realizability of either $D \curlywedge C$ or $Q_{8}$ implies that of $M_{16}$.

Proof. If $s(K)=1$, the obstruction for $M_{16}$ becomes simply $(a, 2 b)$. If $2 \notin \dot{K}^{2}$, letting $b=2$ makes the obstruction trivial. If $2 \in \dot{K}^{2}$, then the obstruction becomes $(a, b)$, which is the same as the obstruction for $D_{8}$. This group is realizable if 
and only if $K$ is not rigid. Now assume $s(K)=2$. Let $a=-1, b \notin \pm \dot{K}^{2}$, and $x=2 b$. Then $(-1,-1)=(-1,2 b)(-1,2 b)=1$, so $M_{16}$ is realizable. Finally assume $s(K) \geq 4$. If $2 \notin \dot{K}^{2}$, then $2,-2$ are independent. Let $a=2, b=-2, x=1$. Then $(2,-1)=(2,-1)(1,-1)=1$, so $M_{16}$ is realizable. If $2 \in \dot{K}^{2}$, we have shown in the proof of Proposition 2.11 that realizability of $C_{4}$ implies the realizability of $M_{16}$, and $C_{4}$ is realizable unless $s(K)=\infty$ and $K$ is pythagorean.

Corollary 2.14. Assume $s(K)=1$. If $2 \in \dot{K}^{2}$, realizability of any of $D_{8}, Q_{8}$, $Q \curlywedge C, D \curlywedge C$ is equivalent to realizability of $M_{16}$. If $2 \notin \dot{K}^{2}$ and $\left|\dot{K} / \dot{K}^{2}\right| \geq 4$, then $M_{16}$ is always realizable.

Proof. This follows directly from the preceding proof and Corollary 2.7.

Proposition 2.15. Realizability of any of $M_{16}, Q_{16}$, or $S D_{16}$ implies realizability of $\mathrm{C}_{4}$.

Proof. First, $C_{4}$ is a quotient of $M_{16}$, so this is a case of trivial realizability. It is not a quotient of either of the other two groups, however, so these results are of more interest. We may assume $s(K)=\infty$ and $K$ is pythagorean, since $C_{4}$ otherwise is always realizable. Thus $2 \in \dot{K}^{2}$. We have observed in this case that $Q_{16}$ is equivalent to $Q_{8}$ (Proposition 2.11) which is equivalent to $Q \curlywedge C$ (Proposition 2.5), which has $C_{4}$ as a quotient, so realizability of $Q_{16}$ implies realizability of $C_{4}$.

Finally, suppose $S D_{16}$ is realizable and $2 \in \dot{K}^{2}$. Then there exist independent $a, b \in \dot{K}$ such that $(a, b)=1$ and $(a,-1)(x,-a b)=1$ for some $x \in \dot{K}$. By the common slot, or linkage, property for quaternion algebras, there exists $y \in \dot{K}$ such that $(a,-1)=(y,-1),(x,-a b)=(y,-a b)$, and $(y, a b)=1$. Thus $(a y,-1)=$ $(a,-1)(y,-1)=1$. If $y \neq a$, then $(a y,-1)=1$ implies the existence of a $C_{4^{-}}$ extension containing $K(\sqrt{a y})$. If $y=a$, then $(a, a b)=1=(a, b)$ implies $(a, a)=1$, so there exists a $C_{4}$-extension containing $K(\sqrt{a})$.

\section{Realizability Results By CONDitions on the Field $K$}

In this section we organize the realizability results according to the level of $K$. For groups of order 16, our results yield or improve the results obtained in [Je:1989], [Je:1992] and [L:1995]. Jensen's results are obtained using a variety of methods, and many hold for larger classes of 2-groups, not just those of order 16 . When considering just groups of order 16 , however, most of these results can be obtained and even strengthened working solely with the obstructions to the realizability of the groups.

Theorem 3.1. Assume $s(K)=1$.

(1) The group $\left(C_{4}\right)^{n}$ is realizable if and only if $\left|\dot{K} / \dot{K}^{2}\right| \geq 2^{n}$.

(2) If any one of the groups $D_{8}, Q_{8}, D \curlywedge C, Q \curlywedge C$ is realizable, so are the rest. If $\left|\dot{K} / \dot{K}^{2}\right| \geq 8$, then realizablility of these groups is equivalent to realizability of DC.

(3) If $2 \notin \dot{K}^{2}$ and $\left|\dot{K} / \dot{K}^{2}\right| \geq 4$, then $M_{16}$ is always realizable.

(4) If any one of the groups $D_{16}, Q_{16}, S D_{16}$ is realizable, then so are the other two. Realizability of any of these groups trivially realizes $D_{8}$. Thus, if $\left|\dot{K} / \dot{K}^{2}\right| \geq 16$, realizability of any of these groups implies realizability of all groups of order 16 .

(5) If $2 \in \dot{K}^{2}$, i.e. if $K$ contains a primitive 8 th root of unity, then realizability of any of the groups $D_{8}, D_{16}, M_{16}$ implies realizability of the others. Thus, if $\left|\dot{K} / \dot{K}^{2}\right| \geq$ 16 and $K$ contains a primitive 8 th root of unity, realizability of any nonabelian group of order 16 implies realizability of all groups of order 16. 
Proof. Most of the results follow directly from the propositions and corollaries in $\S 2$. For (1), observe that $(a,-1)=1$ for all $a \in \dot{K}$. Thus the number of independent $C_{4}$-extensions that exist over $K$ is precisely the number of independent square classes in $K$. (2) follows directly from Corollary 2.7 and (3) from Corollary 2.14. The first statement of (4) follows from Proposition 2.9. The first statement of (5) comes from Corollary 2.10. The final statements of (4) and (5) now follow from the rest of the theorem. Notice that $\left|\dot{K} / \dot{K}^{2}\right| \geq 8$ is sufficient for realizability of all of these groups except $C_{2}^{4}$.

Theorem 3.2. Assume $s(K)=2$.

(1) The group $C_{4}$ is always realizable.

(2) If $\left|\dot{K} / \dot{K}^{2}\right| \geq 4$, the groups $D_{8}, Q_{8}, Q \curlywedge C$, and $M_{16}$ are always realizable.

(3) The group $D_{16}$ is realizable if and only if $Q_{16}$ is.

(4) The group $D \curlywedge C$ is realizable if and only if $C_{4} \times C_{4}$ is.

(5) If $\left|\dot{K} / \dot{K}^{2}\right| \geq 4$ and $2 \in \dot{K}^{2}$, then $D_{16}$ is always realizable, and realizability of $D \curlywedge C$ implies realizability of $S D_{16}$. If $\left|\dot{K} / \dot{K}^{2}\right| \geq 8$, realizability of $S D_{16}$ implies realizability of $D C$.

(6) If $\left|\dot{K} / \dot{K}^{2}\right| \geq 4$ and $-2 \in \dot{K}^{2}$, then $S D_{16}$ is always realizable, and realizability of $D \curlywedge C$ implies realizability of $D_{16}$. If $\left|\dot{K} / \dot{K}^{2}\right| \geq 8$, realizability of $D_{16}$ implies realizability of $D C$.

(7) If $\left|\dot{K} / \dot{K}^{2}\right| \geq 4$ and $2 \notin \pm \dot{K}^{2}$, then the groups $D_{16}, Q_{16}, D \curlywedge C, S D_{16}$, and $C_{4} \times C_{4}$ are always realizable. If $\left|\dot{K} / \dot{K}^{2}\right| \geq 8, D C$ is also realizable. If $\left|\dot{K} / \dot{K}^{2}\right| \geq 16$, every group of order 16 is realizable.

Proof. (1) follows from Proposition 2.1; note in particular that $(-1,-1)=1 \epsilon$ $\mathrm{Br}_{2}(K)$, realizing $C_{4}$. (2) comes from Propositions 2.2, 2.3, 2.5, and 2.13. Proposition 2.11 gives (3). Proposition 2.6 shows the realizability of $D \curlywedge C$ is implied by that of $C_{4} \times C_{4}$. Conversely, if $D \curlywedge C$ is realizable, there exists independent $a, b \in \dot{K}$ with $(a,-1)=(a, b)=1$. If $a \neq-1$, there exist $C_{4}$-extensions containing $K(\sqrt{a})$ and $K(\sqrt{-1})$. If $a=-1$, there exist $C_{4}$-extensions containing $K(\sqrt{b})$ and $K(\sqrt{-1})$. This gives (4). Propositions 2.8 and 2.9 give both (5) and (6).

Finally, for (7), Proposition 2.9 gives realizability of $D_{16}$; this together with Proposition 2.11 gives realizability of $Q_{16}$. The group $C_{4} \times C_{4}$ is realizable because $(-1,-1)=(-1,2)=1$, so there exist independent $C_{4}$-extensions of $K$ containing $K(\sqrt{-1})$ and $K(\sqrt{2})$ respectively. Proposition 2.12 gives the realizability of $S D_{16}$. The realizability of $D C$ follows from Proposition 2.8. Finally, all of these combined with (2) give the last statement.

Theorem 3.3. Assume $s(K) \geq 4$ and $\left|\dot{K} / \dot{K}^{2}\right| \geq 4$.

(1) The groups $D_{8}$ and $D_{16}$ are always realizable.

(2) The group $Q_{8}$ is realizable if and only if $Q \curlywedge C$ is.

(3) If $2 \notin \dot{K}^{2}$, the groups $C_{4}, D \curlywedge C, S D_{16}$, and $M_{16}$ are always realizable. If $\left|\dot{K} / \dot{K}^{2}\right| \geq 8$, then $D C$ is also realizable.

(4) If $2 \in \dot{K}^{2}$, then $Q_{16}$ is realizable if and only if $Q_{8}$ is. If any one of the groups $C_{4}, D \curlywedge C, S D_{16}, M_{16}$ is realizable, so are the rest. All of these groups are automatically realized by $Q_{8}$. If $\left|\dot{K} / \dot{K}^{2}\right| \geq 8$, realizability of any of these groups implies realizability of $D C$. Thus, in this case, realizability of all nonabelian groups of order 16 is implied by realizability of $Q_{8}$. 
Proof. Propositions 2.2 and 2.9 give (1), and (2) is exactly Proposition 2.5. Since $(-1,2)=1 \in B r_{2}(K)$ always, if $2 \notin \dot{K}^{2}, K$ cannot be pythagorean, and since $-2 \notin$ $\dot{K}^{2}$, this also implies that $K$ cannot be rigid. Thus Propositions 2.1, 2.6, 2.12, and 2.13 give (3). The first statement of (4) follows from Proposition 2.11. The second statment can be derived as follows: Propositions 2.12 and 2.13 show realizability of $D \curlywedge C$ imply realizability of $M_{16}$ and $S D_{16}$. Proposition 2.15 shows realizability of either of these groups implies realizability of $C_{4}$. And Proposition 2.6 together with Proposition 2.1 gives the fact that $C_{4}$ realizes $D \curlywedge C$ when $s(K) \geq 4$. The third statement of (4) follows from Proposition 2.3, and the fourth from Proposition 2.4. The final statement summarizes the results above.

We have the following proposition, improving on results of Jensen which state that realizability of $Q_{8}$ implies realizability of $Q \curlywedge C$, implying realizability of $D_{8}$, and realizability of $D_{8}$ implies realizability of one of $D_{16}, S D_{16}$, or $M_{16}$.

Proposition 3.4. Realizability of $Q_{8}$ is equivalent to that of $Q \curlywedge C$ and implies that of $D_{8}$. Realizability of $D_{8}$ implies realizability of at least one of $D_{16}$ or $M_{16}$.

The first result in Proposition 3.4 follows from Proposition 2.5 and Theorems $3.1(2), 3.2(2)$, and 3.3(2). The second result can be derived as follows. Theorem $3.1(3),(5)$ shows that if $s(K)=1, D_{8}$ automatically realizes $M_{16}$. The realizability of $D_{8}$ implies $\left|\dot{K} / \dot{K}^{2}\right| \geq 4$, so if $s(K)=2, M_{16}$ is realizable by Theorem 3.2(2), and if $s(K) \geq 4, D_{16}$ is realizable by Theorem 3.3(1).

Jensen also derives a number of results which depend on square class considerations and existence of certain roots of unity in the field. Again, if one considers just groups of order 16, these results can be obtained from Theorems 3.1-3.3. The roots of unity needed by Jensen in this case are at most 8 th roots of 1 , so considering the behavior of -1 and 2 in $\dot{K} / \dot{K}^{2}$ suffices.

\section{REFERENCES}

[GSS:1995] H. G. Grundman, T. L. Smith and J. Swallow, Groups of order 16 as Galois groups, Expo. Math. 13 (1995), 289-319.

[Je:1989] C. U. Jensen, On the representations of a group as a Galois group over an arbitrary field, Théorie des nombres - Number Theory (J.-M. De Koninck and C. Levesque, eds.), Walter de Gruyter, 1989, pp. 441-458. MR 90k:12006

[Je:1992] ㄴ Finite groups as Galois groups over arbitrary fields, Cont. Math. 131 (1992), 534-448. MR 93i:12008

[KuLe:1975] W. Kuyk and H. W. Lenstra, Jr., Abelian extensions of arbitrary fields, Math. Ann. 216 (1975), 99-104. MR 54:12730

[L:1995] A. Ledet, On 2-groups as Galois groups, Canad. J. Math. 47 (1995), 1253-1273.

[MiSm:1991] J. Mináč and T. L. Smith, A characterization of C-fields via Galois groups, J. Algebra 137 (1991), 1-11. MR 92c:11033

[Wa:1990] R. Ware, A note on the quaternion group as Galois group, Proc. Amer. Math. Soc. 108 (1990), 621-625. MR 90g:12006

[Wh:1957] G. Whaples, Algebraic extensions of arbitrary fields, Duke Math. J. 24 (1957), 201204. MR 19:8b

[Wi:1936] E. Witt, Konstruktion von galoisschen Körpern der Charakteristik $p$ zu vorgegebener Gruppe der Ordnung $p^{f}$, J. Reine Angew. Math. 174 (1936), 237-245.

Department of Mathematics, Bryn Mawr College, Bryn Mawr, Pennsylvania 19010 and Mathematical Sciences Research Institute, Berkeley, California 94720

E-mail address: grundman@brynmawr.edu

Department of Mathematics, University of Cincinnati, Cincinnati, Ohio 45221-0025

E-mail address: tsmith@math.uc.edu 